https://helda.helsinki.fi

\title{
Bing meets Sobolev
}

\section{Onninen, Jani}

2021-03

Onninen , J \& Pankka , P 2021 , ' Bing meets Sobolev ', Revista Matematica

Iberoamericana, vol. 37 , no. 5 , pp. 1897-1916 . https://doi.org/10.4171/RMI/1252

http://hdl.handle.net/10138/337007

https://doi.org/10.4171/RMI/1252

acceptedVersion

Downloaded from Helda, University of Helsinki institutional repository.

This is an electronic reprint of the original article.

This reprint may differ from the original in pagination and typographic detail.

Please cite the original version. 


\title{
BING MEETS SOBOLEV
}

\author{
JANI ONNINEN AND PEKKA PANKKA
}

Dedicated to Pekka Koskela on the occasion of his 59th birthday.

Abstract. We show that, for each $1 \leq p<2$, there exists a wild involution $\mathbb{S}^{3} \rightarrow \mathbb{S}^{3}$ in the Sobolev class $W^{1, p}\left(\mathbb{S}^{3}, \mathbb{S}^{3}\right)$.

\section{INTRODUCTION}

A special case of a classical result of P.A. Smith [31] states that the fixed point set of an involution $\mathbb{S}^{3} \rightarrow \mathbb{S}^{3}$ is homeomorphic to either $\mathbb{S}^{1}$ or $\mathbb{S}^{2}$. More precisely, the fixed point set is homeomorphic to $\mathbb{S}^{2}$ for orientationreversing involutions and homeomorphic to $\mathbb{S}^{1}$ for orientation-preserving (non-identity) involutions. Recall that a homeomorphism $f: \mathbb{S}^{3} \rightarrow \mathbb{S}^{3}$ is an involution if $f \circ f=\mathrm{id}$, that is, $f^{-1}=f$. We call orientation-reversing involutions reflections.

For a $C^{1}$-involution, the fixed point set is a smooth submanifold by a similarly classical result of Bochner [8]. Topological involutions, on the other hand, exhibit much wilder behavior. In a celebrated paper [5] Bing constructed an example of an orientation-reversing wild involution of $\mathbb{S}^{3}$ which has a wildly embedded 2-sphere as its fixed point set. Montgomery and Zippin [25] modified Bing's construction to obtain an orientation-preserving wild involution of $\mathbb{S}^{3}$ having a wildly embedded circle as its fixed point set. Recall that an embedded $k$-sphere $S \subset \mathbb{S}^{3}$ is tamely embedded (or tame for short) if there is a homeomorphism $h: \mathbb{S}^{3} \rightarrow \mathbb{S}^{3}$ for which $h(S)=\mathbb{S}^{k} \subset \mathbb{S}^{3}$, and wildly embedded otherwise.

In what follows, we say than an involution $\mathbb{S}^{3} \rightarrow \mathbb{S}^{3}$ is tame (resp. wild) if its fixed point set is tame (resp. wild). In this terminology $C^{1}$-involutions of $\mathbb{S}^{3}$ are tame. In the orientation-reversing case, this is a straight forward consequence of the collarability of smooth 2 -spheres in $\mathbb{S}^{3}$ and the generalized Schoenflies theorem [10]. The case of orientation-preserving involutions is a part of the Smith conjecture, proved first by Waldhausen [32] in the special case of diffeomorphisms of order 2 and Morgan and Bass [26] in the general case. Recall that in two dimensions involutions of $\mathbb{S}^{2}$ are tame; see Brouwer $[9]$.

Date: April 9, 2020.

2010 Mathematics Subject Classification. Primary 57S25; Secondary 57R12, 57N45, 46E35, 30C65.

Key words and phrases. Fixed point set, Sobolev homeomorphism, wild involution.

This work was supported in part by the Academy of Finland project \#297258 and the NSF grant DMS-1700274. 
It is clear from the setting that Bochner's result on the smoothness of the fixed point set does not extend below $C^{1}$-smoothness of the involution. Indeed, it suffices to conjugate $\lambda:\left(x_{1}, x_{2}, x_{3}\right) \mapsto\left(-x_{1}, x_{2}, x_{3}\right)$ by the non-smooth bilipschitz homeomorphism $h: \mathbb{R}^{3} \rightarrow \mathbb{R}^{3},\left(x_{1}, x_{2}, x_{3}\right) \mapsto$ $\left(x_{1}+\left|x_{2}\right|, x_{2}, x_{3}\right)$, to obtain an involution $f=h \circ \lambda \circ h^{-1}$ of $\mathbb{R}^{3}$ for which the fixed point set $h\left(\{0\} \times \mathbb{R}^{2}\right)$ is not a smooth submanifold of $\mathbb{R}^{3}$. Conjugation of $f$ by the stereographic projection and extending the obtain homeomorphism to $e_{4} \in \mathbb{S}^{3}$ yields now an example of the desired type.

The question, whether the fixed point set is wildly embedded under weaker assumptions on smoothness, has no such easy answer. In [19] Heinonen and Semmes asked (Question 26) whether there exists a wild quasiconformal reflection of $\mathbb{S}^{3}$; the answer to this question is open even in the case of bilipschitz involutions of $\mathbb{S}^{3}$. ${ }^{1}$

In this article we consider the Sobolev regularity of wild involutions related to Bing's construction [5]. Bing's construction has been used to obtain several others wild constructions in quasiconformal geometry, most notably by Freedman and Skora [16] for wild quasiconformal actions and by Semmes [29] for quasisymmetric non-parametrization theorems; see also Heinonen$\mathrm{Wu}[20]$ and $[27,28]$ for similar results in dimensions $n>3$. However, to our knowledge the regularity of wild involutions have not been considered in the literature apart from the modulus of continuity; see Bing [7]. Our main result reads as follows.

Theorem 1.1. For $p \in[1,2)$ there exist an orientation-preserving and an orientation-reserving wild involution of $\mathbb{S}^{3}$ in the Sobolev space $W^{1, p}\left(\mathbb{S}^{3}, \mathbb{S}^{3}\right)$.

As mentioned, our constructions are Sobolev space versions of the constructions of Bing and Montgomery-Zippin. The wild involution $f: \mathbb{S}^{3} \rightarrow \mathbb{S}^{3}$ is a quotient of a linear involution $\iota: \mathbb{S}^{3} \rightarrow \mathbb{S}^{3}$

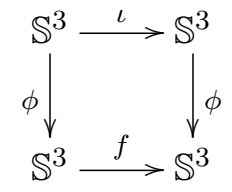

in a monotone map $\phi: \mathbb{S}^{3} \rightarrow \mathbb{S}^{3}$ associated to the Cantor set Bing's double in [5]. In the orientation-reversing case, the isometric involution $\iota$ is $\left(x_{1}, x_{2}, x_{3}, x_{4}\right) \mapsto\left(-x_{1}, x_{2}, x_{3}, x_{4}\right)$ and the fixed point set is a wild 2 -sphere. In the orientation-preserving case, the corresponding involution $\iota$ is the reflection $\left(x_{1}, x_{2}, x_{3}, x_{4}\right) \mapsto\left(-x_{1},-x_{2}, x_{3}, x_{4}\right)$ and the fixed point set is a wild 1-sphere.

To obtain a Sobolev regular wild involution, we consider a modified version of the defining sequence for Bing's double. We show that there is a defining sequence, consisting of solid 3-tori, which are uniformly bilipschitz to certain model cubical 3 -tori in their inner metric. We use here similar cubical straightening methods as in [15]. This allows us to compute the derivative of $f$ from the homeomorphisms in Bing's shrinking process [7] in the complement of the Bing's double. For a given $p \in[1,2)$, we obtain the

\footnotetext{
${ }^{1}$ It is announced in [18] that quasiconformal reflections are tame.
} 
$L^{p}$-integrability of $D f$ by considering a defining sequence consisting of thin solid tori having sufficiently small total volume on each level.

Heuristically, the exponent $p=2$ is associated to the balance between volume of the solid tori and the local bilipschitz constant of homeomorphisms rotating the interiors of the tori in the defining sequence.

We do not know whether our method yields the $W^{1,2}$-Sobolev regularity for $f$. Note, however, that Theorem 1.1 yields the integrability of the adjoint $D^{\#} f$ of $D f$. Namely, it follows from the change of variables formula that

$$
\int_{\mathbb{S}^{3}}|D f(x)|^{p} \mathrm{~d} x=\int_{\mathbb{S}^{3}} \frac{\left|D^{\#} f(x)\right|^{p}}{J_{f}(x)^{p-1}} \mathrm{~d} y
$$

for $p \in[1,2)$, where $J_{f}$ is the Jacobian determinant of the differential $D f$. In spirit of the question of Heinonen and Semmes, it would be interesting to know whether there exist wild involutions in $W^{1, p}\left(\mathbb{S}^{3}, \mathbb{S}^{3}\right)$ for $p \geq 2$.

During the course of the proof of Theorem 1.1, we obtain that the monotone map $\phi: \mathbb{S}^{3} \rightarrow \mathbb{S}^{3}$ is in the same Sobolev space $W^{1, p}$ as the involution $f: \mathbb{S}^{3} \rightarrow \mathbb{S}^{3}$. It should, however, be noted that the Sobolev regularity of $f$ does not follow immediately from the Sobolev regularity of $\phi$ since a priori the composition $\phi \circ \iota \circ \phi^{-1}$, in the complement of the singular set, is not in $W^{1,1}$. An additional cancellation property of $f$ is needed to prove the seeked regularity.

Connection to nonlinear elasticity. Our original interest to investigate the Sobolev regularity of homeomorphisms comes from the theory of nonlinear elasticity - in particularly the Ball-Evans approximation problem [4]. It asks if a $W^{1, p}$-Sobolev homeomorphism can be approximated in the strong topology of $W^{1, p}$ by piecewise affine invertible mappings. J. M. Ball attributes this question to L. C. Evans and points out its relevance to the regularity of minimizers of neohookean energy functionals. In the context of nonlinear elasticity $[1,3,13]$, one typically deals with two or three dimensional models. The Ball-Evans problem is completely understood in the planar case $[21,23]$ and wildly open in dimension three.

It is worth noting that an important result in the Ball-Evans problem in dimension $n=4$ is due to Hencl and Vejnar [22] for $W^{1,1}$-homeomorphisms and in dimensions $n \geq 4$ by Campbell, Hencl, and Tengvall [12]: for each $1 \leq p<[n / 2]$, there is a $W^{1, p}$-Sobolev homeomorphism, which cannot be approximated by piecewise affine homeomorphisms. Here $[a]$ denotes the integer part of $a$.

The connection between wild involutions and the Ball-Evans problem is via cellular mappings. We finish this introduction by discussing this connection between geometric topology and nonlinear elasticity.

To build a viable theory of minimization problems for three or higher dimensional models, we come to the question on enlargement of the class Sobolev homeomorphisms. Clearly, enlarging the set of the admissible mappings may change the nature of the energy-minimal solutions. In two dimensions, the classical Youngs approximation theorem [33] states that a continuous map between 2-spheres is monotone if and only if it is a uniform limit of homeomorphisms. Using a Sobolev variant of Youngs approximation theorem [24], we may enlarge the minimization to monotone mappings and 
to avoid the Lavrentiev phenomenon, that is, the avoid the change of the infimum energy in the enlargement of the class of admissible maps.

In three dimensions it is possible to construct monotone mappings of the 3 -sphere onto itself which cannot be uniformly approximated by homeomorphisms [6]. However, the approximation is possible for cellular mappings; mappings whose inverse image of a point is an intersection of a decreasing sequence of $n$-cells, the notion introduced by Brown [11]. The monotone map $\phi$ obtained in the construction of the Bing's double is an example of a cellular mapping.

Armentrout showed [2] that cellular mappings of an 3-manifold onto itself can be approximated by homeomorphisms; see also Siebenmann [30]. We refer to a book of Daverman [14] for the development of these mappings as a part of the theory of decomposition spaces and manifold recognition problems.

A Sobolev variant of the result of Armentrout would convince us that the Lavrentiev phenomenon in three dimensional minimization problems can be avoided by adopting Sobolev cellular mappings. Nevertheless the theory of Sobolev cellular mappings is still in its infancy.

Acknowledgements Authors thank Piotr Hajłasz [17] and Pekka Koskela [17] for inspiration regarding the title. We also thank the referee for comments drasitically improving the manuscript.

\section{Cubical PREliminaries}

Let $\mathcal{D}$ be the collection of dyadic cubes in $\mathbb{R}^{3}$ of side length at most 1 ; that is, cubes $Q=2^{-k}\left(v+[0,1]^{3}\right)$, where $v \in \mathbb{Z}^{3}$ and $k \in \mathbb{Z}_{+}$. Given a subcollection $C \subset \mathcal{D}$, we denote their union $|C|=\bigcup C$. We call the number of cubes \#C in $\mathrm{C}$ the cubical length of $\mathrm{C}$.

We say that cubes $Q$ and $Q^{\prime}$ in $\mathcal{D}$ are adjacent if $Q \cap Q^{\prime}$ is a common face of both cubes. In particular, adjacent cubes have the same side length. We also say that cubes $Q$ and $Q^{\prime}$ meet if $Q \cap Q^{\prime} \neq \varnothing$.



Figure 1. A collection $\mathrm{C}$ of five adjacent cubes and their adjacency graph $\Gamma(\mathrm{C})$.

Given a collection $\mathrm{C}$ of cubes $\mathcal{D}$ of the same side length, the adjacency graph $\Gamma(\mathrm{C})$ is the graph having cubes $Q$ in $\mathrm{C}$ as vertices and pairs $\left\{Q, Q^{\prime}\right\}$ of adjacent cubes $Q$ and $Q^{\prime}$ in $\mathrm{C}$ as edges; see Figure 1 for an example of an adjacency graph. For each $Q \in \mathrm{C}$, we denote $\mathcal{N}_{\mathrm{C}}(Q)$ the collection of all cubes in $\mathrm{C}$ which intersect $Q$. We call $\mathcal{N}_{\mathrm{C}}(Q)$ the cubical neighborhood of $Q$ in $\mathrm{C}$. Note that $\mathcal{N}_{\mathrm{C}}(Q)$ contains all cubes adjacent to $Q$, but may contain 
also other cubes. We also denote $\sigma(\mathrm{C})$ the common side length of the cubes in $\mathrm{C}$.

2.1. Cubical loops and arcs. In what follows, we consider mainly cubical arcs and loops, which are collactions of cubes $C$ for which the graph $\Gamma(\mathrm{C})$ has valence at most two. For the definitions of cubical loops and arcs, we distinguish first three special classes of cubes.

Definition 2.1. A cube $Q \in \mathrm{C}$ is an $I$-cube if $Q$ is adjacent to exactly two cubes $Q_{+}$and $Q_{-}$in $C$ and $Q_{+} \cap Q_{-}=\varnothing$.

Here the heuristic idea is that the union $Q_{+} \cup Q \cup Q_{-}$is an image of $[0,1]^{2} \times[0,3]$ under a similarity map.

Definition 2.2. A cube $Q \in \mathrm{C}$ is a corner of $\mathrm{C}$ if $Q$ is (again) adjacent to exactly two cubes $Q_{+}$and $Q_{-}$in $\mathrm{C}$ and $Q_{+} \cap Q_{-} \neq \varnothing$.

Now the heuristic idea is that the union $Q_{+} \cup Q \cup Q_{-}$is an image of the union of $\left(e_{1}+[0,1]^{3}\right) \cup[0,1]^{3} \cup\left(e_{2}+[0,1]^{3}\right)$ under a similarity map. For illustrations of an $I$-cube and a corner, see Figure 2. We denote $\mathcal{I}(\mathrm{C})$ and Corner $(\mathrm{C})$ the collections of $I$-cubes and corners of $\mathrm{C}$, respectively.

Finally, we define terminal cubes of a collection.

Definition 2.3. A cube $Q \in \mathrm{C}$ is a terminal cube of $\mathrm{C}$ if $Q$ is a leaf of the adjacency graph $\Gamma(\mathrm{C})$, that is, $Q$ is adjacent to exactly one cube in $\mathrm{C}$.
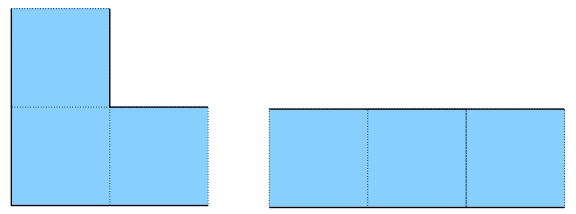

FiguRE 2. A corner and a segment.

Having these three definitions at our disposal, we may define cubical loops and cubical arcs.

Definition 2.4. A finite collection $\mathrm{L}$ of dyadic cubes is a cubical loop if the cubes in $\mathrm{L}$ have the same side length, each cube in $\mathrm{L}$ is either an $I$-cube or a corner, and cubical neighborhoods of corners of $L$ are mutually disjoint. A loop $\mathrm{L}$ is a model loop if it has exactly four corners.

Note that, by finiteness of $L$, the adjacency graph $\Gamma(L)$ of a cubical loop $\mathrm{L}$ is always a cycle; see Figure 3 for an example. In particular, $|\mathrm{L}|$ is homeomorpic to the solid 3 -torus $\bar{B}^{2} \times \mathbb{S}^{1}$.

Definition 2.5. A finite collection A of dyadic cubes is a cubical arc if the cubes in $A$ have the same side length, $A$ has two terminal cubes, each other cube in $\mathrm{A}$ is either an $I$-cube or a corner, and cubical neighborhoods of corners of A are mutually disjoint. A cubical arc is a segment if it has no corners. 



Figure 3. A cubical loop and a model loop of the same cubical length.

Remark. We would like to emphasize a technical point. In what follows, we consider cubical loops and arcs primarily as combinatorial objects. In particular, we keep distinguishing a cubical loop $L$ from its union $|\mathrm{L}|$. Formally, $\mathrm{L}$ is a collection of cubes in $\mathbb{R}^{3}$, whereas the union $|\mathrm{L}|=\bigcup_{Q \in \mathrm{L}} Q$ is a subset in $\mathbb{R}^{3}$.

Remark. We note also that cubical arcs admit an alternative characterization. A finite collection $A$ of dyadic cubes of the same side length is a cubical arc if and only if $|\mathrm{A}|$ is an 3-cell and there exists a linear order $Q_{1}, \ldots, Q_{k}$ of cubes in $\mathrm{A}$, where $k=\# \mathrm{~A}$ is the number of cubes in $\mathrm{A}$, so that $Q_{j}$ is adjacent to $Q_{j+1}$ for each $j \in\{1, \ldots, k-1\}$.

The fact, which will play a crucial role in the forthcoming discussion, is that - in its inner geometry - a cubical arc is locally uniformly bilipschitz equivalent to a segment. Clearly, cubical arcs do not have this property globally uniformly with respect to the ambient geometry of $\mathbb{R}^{3}$. We formulate this precisely in Lemma 2.6 after introducing some terminology for the statement.

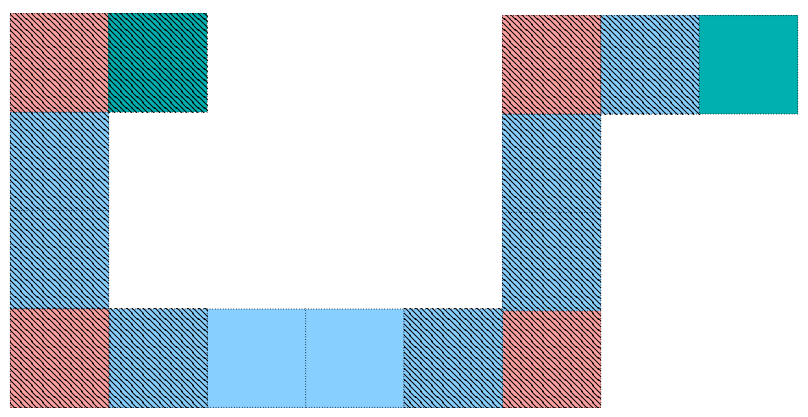

Figure 4. A cubical arc; corner cubes in pink, $I$-cubes in light blue, and terminal cubes in cyan, cubical neighborhoods of corners shaded.

For the statement of bilipschitz equivalence of cubical arcs, we introduce the notion of a symmetry plane. Let $Q$ be a cube in an arc A which is not 
a terminal cube of $\mathrm{A}$. Then $\mathcal{N}_{\mathrm{A}}(Q)$ consists of three cubes and there exists a unique affine hyperplane (of codimension 1) $P_{\mathrm{A}}(Q)$ in $\mathbb{R}^{3}$ which divides $\left|\mathcal{N}_{\mathrm{A}}(Q)\right|$ into two congruent 3-cells. We call $P_{\mathrm{A}}(Q)$ the symmetry plane of $Q$ (with respect to A); see Figure 5 for a symmetry plane for a corner. We use the same terminology and notation also in the case of cubical loops.

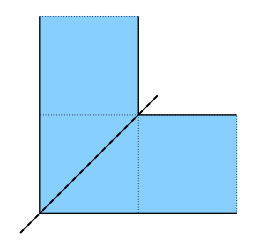

FiguRe 5. Symmetry plane of a corner.

Lemma 2.6. There exists an absolute constant $L_{\text {inner }} \geq 1$ with the following property: Let $\mathrm{A}$ be a cubical arc in $\mathbb{R}^{3}$, and $\mathrm{S}$ a segment having the same number of cubes as $\mathrm{A}$ and having cubes of the same side length than $\mathrm{A}$. Let also $\gamma: \Gamma(\mathrm{A}) \rightarrow \Gamma(\mathrm{S})$ be a graph isomorphism. Then there exists a homeomorphism $\varphi_{\mathrm{A}}^{\mathrm{S}}:|\mathrm{A}| \rightarrow|\mathrm{S}|$ having the following properties:

(1) for each $Q \in \mathrm{A}$, the restriction $\left.\varphi_{\mathrm{A}}^{\mathrm{S}}\right|_{\left|\mathcal{N}_{\mathrm{A}}(Q)\right|}:\left|\mathcal{N}_{\mathrm{A}}(Q)\right| \rightarrow\left|\mathcal{N}_{\mathrm{S}}(\gamma(Q))\right|$ is a well-defined homeomorphism,

(2) for each $I$-cube $Q \in \mathrm{A}$, which is not adjacent to a corner in $\mathrm{A}$, the restriction $\left.\varphi_{\mathrm{A}}^{\mathrm{S}}\right|_{Q}: Q \rightarrow \gamma(Q)$ is an isometry,

(3) for a corner $Q \in \mathrm{A}$, the restriction $\left.\varphi_{\mathrm{A}}^{\mathrm{S}}\right|_{\left|\mathcal{N}_{\mathrm{A}}(Q)\right|}:\left|\mathcal{N}_{\mathrm{A}}(Q)\right| \rightarrow\left|\mathcal{N}_{\mathrm{S}}(\gamma(Q))\right|$ is $L_{\text {inner-bilipschiptz, and }}$

(4) for each $Q \in \mathrm{A}$, which is not a terminal cube in $\mathrm{A}$, the restriction $\left.\varphi_{\mathrm{A}}^{S}\right|_{P_{\mathrm{A}}(Q) \cap Q}: P_{\mathrm{A}}(Q) \cap Q \rightarrow P_{\mathrm{S}}(\gamma(Q)) \cap \gamma(Q)$ is well-defined and affine.

Proof. It suffices to consider the special case that $\# \mathrm{~A}=3$ and $\mathrm{A}$ has a corner. In this case, we may assume that $|\mathrm{A}|$ is the 3 -cell $D \times[0,1]$, where

$$
D=([0,1] \times[1,2]) \cup[0,1]^{2} \cup([1,2] \times[0,1]) .
$$

Let now $\mathrm{S}$ be a straight arc of three cubes. Again, we may assume that $|\mathrm{S}|=E \times[0,1]$, where $E=([-1,0] \times[0,1]) \cup[0,1]^{2} \cup([1,2] \times[0,1])$; see Figure 6 .
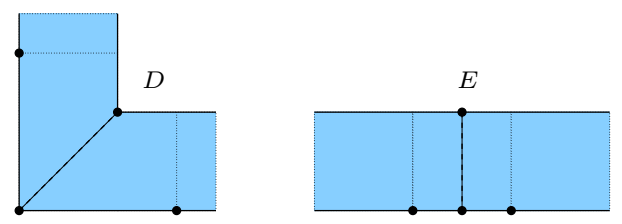

Figure 6. Cells $D$ and $E$.

Then, clearly, there exists a bilipschitz homeomorphism $\varphi: D \rightarrow E$ for which

(1) $\left.\varphi\right|_{\{2\} \times[0,1]}=\mathrm{id}$ and

(2) $\left.\varphi\right|_{[0,1] \times\{2\}}:[0,1] \times\{2\} \rightarrow\{-1\} \times[0,1]$ is an isometry. 
Now $\varphi \times$ id: $D \times[0,1] \rightarrow E \times[0,1]$ satisfies the requirements of the claim.

The general case of a cubical arc A now follows by straightening all neighborhoods of corners in A by copies of the map $\varphi$ and extending isometrically over all reduced $I$-blocks using the property (2).

Remark. Since the proof of Lemma 2.6 is local, the claim holds also for loops and model loops of the same cubical length after obvious changes. We omit the details.

For the forthcoming discussion, we give the following definition in sprit of Lemma 2.6. In what follows, we use this definition for graph isomorpisms and graph coverings in the case of cubical arcs, loops, and lines.

Definition 2.7. Let $C$ and $C^{\prime}$ be cubical arcs, loops, or lines, and let $\gamma: \Gamma(C) \rightarrow$ $\Gamma\left(\mathrm{C}^{\prime}\right)$ be a map of graphs. A map $h:|\mathrm{C}| \rightarrow\left|\mathrm{C}^{\prime}\right|$ is a realization of $\gamma$ if

(1) for each $Q \in \mathrm{C}$, the restriction $\left.h\right|_{\left|\mathcal{N}_{\mathrm{C}}(Q)\right|}:\left|\mathcal{N}_{\mathrm{C}}(Q)\right| \rightarrow\left|\mathcal{N}_{\mathrm{C}^{\prime}}(\gamma(Q))\right|$ is a well-defined homeomorphism,

(2) for each $I$-cube $Q \in \mathrm{C}$, which is not adjacent to a corner in C, the restriction $\left.h\right|_{Q}: Q \rightarrow \gamma(Q)$ is an isometry,

(3) for a corner $Q \in \mathrm{C}$, the restriction $\left.h\right|_{\left|\mathcal{N}_{\mathrm{C}}(Q)\right|}:\left|\mathcal{N}_{\mathrm{C}}(Q)\right| \rightarrow\left|\mathcal{N}_{\mathrm{C}^{\prime}}(\gamma(Q))\right|$ is $L_{\text {inner-bilipschiptz, and }}$

(4) for each $Q \in \mathrm{C}$, which is not a terminal cube in C, the restriction $\left.h\right|_{P_{\mathrm{C}}(Q) \cap Q}: P_{\mathrm{C}}(Q) \cap Q \rightarrow P_{\mathrm{C}^{\prime}}(\gamma(Q)) \cap \gamma(Q)$ is well-defined and affine.

\section{NeSted LOOPS AND TWIST MAPS}

In this section we consider pairs $\left(L, L^{\prime}\right)$ of cubical loops $L$ and $L^{\prime}$ for which $\left|\mathrm{L}^{\prime}\right| \subset \operatorname{int}|\mathrm{L}|$ and the pair $\left(|\mathrm{L}|,\left|\mathrm{L}^{\prime}\right|\right)$ is homeomorphic to the pair $\left(\bar{B}^{2} \times \mathbb{S}^{1}, \bar{B}^{2}(1 / 2) \times \mathbb{S}^{1}\right)$. We also consider particular self-homeomorphisms $\theta:\left(|\mathrm{L}|,\left|\mathrm{L}^{\prime}\right|\right) \rightarrow\left(|\mathrm{L}|,\left|\mathrm{L}^{\prime}\right|\right)$ of pairs which are identity on the boundary of $|\mathrm{L}|$ and, in an heuristic sense, rotate the inner cubical loop $L^{\prime}$. Before giving a precise definitions, we describe a round model for these self-homeomorphisms.

3.1. Twisting in a round model pair. Let $0<r<R$ and $\ell>0$, and consider the solid tori $T(R ; \ell)=\bar{B}^{2}(R) \times \mathbb{S}^{1}(\ell)$ and $T(r ; \ell)=\bar{B}^{2}(r) \times$ $\mathbb{S}^{1}(\ell)$. Now $(T(R ; \ell), T(r ; \ell))$ is a pair of solid 3-tori homeomorphic to $\left(\bar{B}^{2} \times\right.$ $\left.\mathbb{S}^{1}, \bar{B}^{2}(1 / 2) \times \mathbb{S}^{1}\right)$. Let now $\alpha \in[0,2 \pi)$ be an angle. Let also $u_{r, R}: \bar{B}^{2}(R) \rightarrow$ $[0, \alpha]$ be the function

$$
x \mapsto \begin{cases}\alpha, & |x| \leq r \\ \alpha \frac{R-|x|}{R-r}, & 0 \leq|x| \leq R\end{cases}
$$

and $h_{r, R ; \ell}^{\alpha}: T(R ; \ell) \rightarrow T(R ; \ell)$ be the homeomorphism

$$
(x, z) \mapsto\left(x, e^{i u_{r, R}(x)} z\right) .
$$

Then $h_{r, R ; \ell}^{\alpha}$ is a homeomorphism of pairs

$$
h_{r, R ; \ell}^{\alpha}:(T(R ; \ell), T(r ; \ell)) \rightarrow(T(R ; \ell), T(r ; \ell)),
$$

which is the identity on the boundary $\partial T(R ; \ell)$ of $T$ and a rotation, by angle $\alpha$, on the solid torus $T(r ; \ell)$. Metrically, $h_{r, R ; \ell}^{\alpha}$ is bilipschitz with a constant $L \geq 1$ depending only on the angle $\alpha$ and the ratio $R / r$. 
3.2. Nested loops. We describe now the cubical versions of the round model pair $(T(R ; \ell), T(r ; \ell))$ and twisting homeomorphisms $h_{r, R ; \ell}^{\alpha}$. We begin with nested cubical loops and define after that the cubical twist maps.

Definition 3.1. A pair $\left(\mathrm{L}, \mathrm{L}^{\prime}\right)$ of cubical loops is a nested pair if

(1) $\left|\mathrm{L}^{\prime}\right| \subset \operatorname{int}|\mathrm{L}|$,

(2) $I$-cubes of $\mathrm{L}$ contain only $I$-cubes of $\mathrm{L}^{\prime}$,

(3) each corner of $\mathrm{L}$ contains exactly one corner of $\mathrm{L}^{\prime}$,

(4) for each corner $Q$ of $\mathrm{L}$ and the unique corner $Q^{\prime}$ of $\mathrm{L}^{\prime}$ contained in $Q$, have the same symmetry plane, that is, $P_{\mathrm{L}}(Q)=P_{\mathrm{L}^{\prime}}\left(Q^{\prime}\right)$.

We say the nested pair $\left(L, L^{\prime}\right)$ is uniform if

$$
\operatorname{dist}\left(\left|\mathbf{L}^{\prime}\right|, \partial|\mathrm{L}|\right) \geq \sigma\left(\mathrm{L}^{\prime}\right)
$$

where $\sigma\left(\mathrm{L}^{\prime}\right)$ is the common side length of cubes in $\mathrm{L}^{\prime}$.

See Figure 7 for an illustration of a nested pair.

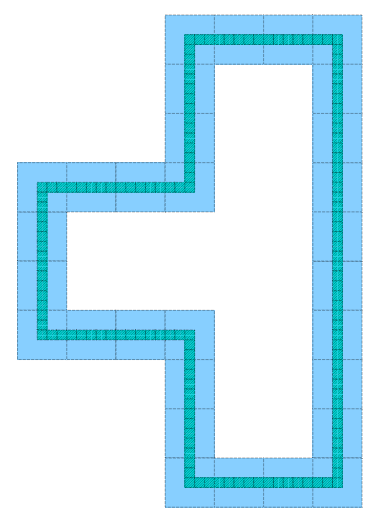

Figure 7. A nested pair.

3.2.1. Rotations of ordered nested pairs. Let $\left(\mathrm{L}, \mathrm{L}^{\prime}\right)$ be a pair of nested loops. Then there exists a natural map $\iota: \mathrm{L}^{\prime} \rightarrow \mathrm{L}$ induced by inclusion of the cubes in $\mathrm{L}^{\prime}$ into cubes in $\mathrm{L}$, that is, for each $Q^{\prime} \in \mathrm{L}^{\prime}$ the image $\iota\left(Q^{\prime}\right)$ is the unique cube in $\mathrm{L}$ containing $Q^{\prime}$.

Given an order $<_{\mathrm{L}}$ for cubes in $\mathrm{L}$, there exists a unique cyclic order $<_{\mathrm{L}^{\prime}}$ for cubes in $L^{\prime}$ for which the map $\iota$ is order preserving. Therefore, we may consider each nested pair as an ordered pair in this sense.

Let now $\mathscr{P}=\left(\mathrm{L}, \mathrm{L}^{\prime}\right)$ be an ordered nested pair. We call an orientation preserving graph isomorphism $\rho: \Gamma\left(\mathrm{L}^{\prime}\right) \rightarrow \Gamma\left(\mathrm{L}^{\prime}\right)$ a rotation of $\mathrm{L}^{\prime}$ in $\mathrm{L}$.

3.3. Twist maps. We define now self-homeomorphisms of pairs $\left(|\mathrm{L}|,\left|\mathrm{L}^{\prime}\right|\right)$ for nested cubical loops. Heuristically, these homeomorphisms correspond to self-homeomorphisms $h_{r, R ; \ell}^{\alpha}$ of the round solid tori pairs.

Definition 3.2. A homeomorphism $h:\left(|\mathrm{L}|,\left|\mathrm{L}^{\prime}\right|\right) \rightarrow\left(|\mathrm{L}|,\left|\mathrm{L}^{\prime}\right|\right)$ is a $\rho$-twist for a rotation $\rho: \Gamma\left(\mathrm{L}^{\prime}\right) \rightarrow \Gamma\left(\mathrm{L}^{\prime}\right)$ if $\left.h\right|_{\partial|\mathbf{L}|}=$ id and $\left.h\right|_{\left|\mathrm{L}^{\prime}\right|}:\left|\mathrm{L}^{\prime}\right| \rightarrow\left|\mathrm{L}^{\prime}\right|$ is a realization of $\rho$. 
The main result of this section is the following lemma. For the statement, let $R_{ \pm}: \mathbb{R}^{3} \rightarrow \mathbb{R}^{3}$ be the linear involution $\left(x_{1}, x_{2}, x_{3}\right) \mapsto\left(x_{1}, \pm x_{2},-x_{3}\right)$. We say that a nested pair $\mathscr{P}=\left(\mathrm{L}, \mathrm{L}^{\prime}\right)$ is $R_{ \pm}$-symmetric if the maps $\mathrm{L} \rightarrow \mathrm{L}$, $Q \mapsto R_{ \pm}(Q)$, and $\mathrm{L}^{\prime} \rightarrow \mathrm{L}^{\prime}, Q^{\prime} \mapsto R_{ \pm}\left(Q^{\prime}\right)$, are well-defined bijections.

Lemma 3.3. There exists a constant $L \diamond \geq 1$ with the following property: Let $\mathscr{P}=\left(\mathrm{L}, \mathrm{L}^{\prime}\right)$ be a uniform nested pair symmetric with respect to $R_{ \pm}$and let $\rho: \mathrm{L}^{\prime} \rightarrow \mathrm{L}^{\prime}$ be a rotation. Then there exists a $\rho$-twist $h_{\rho}:\left(|\mathrm{L}|,\left|\mathrm{L}^{\prime}\right|\right) \rightarrow$ $\left(|\mathrm{L}|,\left|\mathrm{L}^{\prime}\right|\right)$ for which the involution

$$
h_{\rho} \circ R_{ \pm} \circ h_{\rho}^{-1}:\left(|\mathrm{L}|,\left|\mathrm{L}^{\prime}\right|\right) \rightarrow\left(|\mathrm{L}|,\left|\mathrm{L}^{\prime}\right|\right)
$$

is locally $L_{\diamond}\left(\# \mathrm{~L}^{\prime}\right)$-bilipschitz.

Proof. By the method of Lemma 2.6, there exists an absolute constant $L \geq 1$ and an $L$-bilipschitz homeomorphism $h_{\rho}^{\prime}:\left|\mathrm{L}^{\prime}\right| \rightarrow\left|\mathrm{L}^{\prime}\right|$ realizing the rotation $\rho$. We extend $h_{\rho}^{\prime}$ to a $\rho$-twist $h_{\rho}:|\mathrm{L}| \rightarrow|\mathrm{L}|$ as follows. For the extension we fix some auxiliary mappings.

Let $\widetilde{L}$ be the cubical line

$$
\widetilde{\mathrm{L}}=\left\{\sigma(\mathrm{L})\left(k e_{1}+[0,1]^{3}\right) \subset \mathbb{R}^{3}: k \in \mathbb{Z}\right\}
$$

in $\mathbb{R}^{3}$.

We fix in $\widetilde{L}$ the natural order induced by $\mathbb{Z}$ and let $\phi: \widetilde{L} \rightarrow L$ be a natural cubical covering map, that is, an order preserving local injection $\Gamma(\widetilde{L}) \rightarrow$ $\Gamma(\mathrm{L})$. Since $\mathrm{L}$ is a loop, we have that

$$
\phi\left(Q+(\# \mathbf{L}) e_{1}\right)=\phi(Q) .
$$

Using the method of Lemma 2.6 again, we may now fix a locally $L$ bilipschitz covering map $g_{\phi}:|\widetilde{\mathrm{L}}| \rightarrow|\mathrm{L}|$ realizing $\phi$. We may assume that $g_{\phi}$ is $(\# \mathbf{L}) e_{1}$-periodic in the sense that, for every $x \in|\widetilde{\mathbf{L}}|$, we have that

$$
g_{\phi}\left(x+(\# \mathrm{~L}) e_{1}\right)=\phi(x) .
$$

Since $\left(L, L^{\prime}\right)$ is a nested pair, we may, by condition (2) in the definition of nested pairs, assume that there exists a vector $v \in \mathbb{R}^{3}$ for which the infinite cubical line

$$
\widetilde{\mathrm{L}^{\prime}}=\left\{\sigma(\mathrm{L})\left(k e_{1}+[0,1]^{k}+v\right) \subset \mathbb{R}^{3}: k \in \mathbb{Z}\right\}
$$

in $\mathbb{R}^{3}$ has the property that there exists a natural cubical covering map $\phi^{\prime}: \widetilde{\mathrm{L}^{\prime}} \rightarrow \mathrm{L}^{\prime}$ for which the restriction $\left.g_{\phi}\right|_{\left|\widetilde{\mathrm{L}^{\prime}}\right|}$ is a realization of $\phi^{\prime}$, that is,

for each $\tilde{Q}^{\prime} \in \widetilde{\mathrm{L}^{\prime}}$.

$$
g_{\phi}\left(\mathcal{N}_{\tilde{\mathbf{L}^{\prime}}}\left(\tilde{Q}^{\prime}\right)\right)=\mathcal{N}_{\widetilde{\mathbf{L}^{\prime}}}\left(\phi^{\prime}\left(\tilde{Q}^{\prime}\right)\right)
$$

Let now $\tilde{h}_{\rho}^{\prime}:\left|\widetilde{\mathrm{L}^{\prime}}\right| \rightarrow\left|\widetilde{\mathrm{L}^{\prime}}\right|$ be a lift of $h_{\rho}^{\prime}$ in the covering map $g_{\phi}$. To define an extension $|\widetilde{\mathrm{L}}| \rightarrow|\widetilde{\mathrm{L}}|$ of $\tilde{h}_{\rho}^{\prime}$, we give some preliminary definitions.

Recall that $|\widetilde{\mathrm{L}}|=\mathbb{R} \times[0, \sigma(\mathrm{L})]^{2}$ and $\left|\widetilde{\mathrm{L}^{\prime}}\right|=\mathbb{R} \times\left(v+\left[0, \sigma\left(\mathrm{L}^{\prime}\right)\right]^{2}\right)$, where squares $[0, \sigma(\mathrm{L})]^{2}$ and $v+\left[0, \sigma\left(\mathrm{L}^{\prime}\right)\right]^{2}$ are concentric squares in $\mathbb{R}^{2}$. Let now $s:[0, \sigma(\mathrm{L})]^{2} \rightarrow v+\left[0, \sigma\left(\mathrm{L}^{\prime}\right)\right]^{2}$ be the unique scaling mapping corners to corners, and let

$$
S=\mathrm{id} \times s:|\mathrm{L}| \rightarrow\left|\mathrm{L}^{\prime}\right| .
$$

Then $S$ is a bilipschitz homeomorphism with a constant depending only on the ratio $\sigma(\mathrm{L}) / \sigma\left(\mathrm{L}^{\prime}\right)$. 
We fix now a particular lift

$$
\iota: \partial|\widetilde{\mathrm{L}}| \rightarrow \partial|\widetilde{\mathrm{L}}|
$$

of the identity map id: $\partial|\mathrm{L}| \rightarrow \partial|\mathrm{L}|$ as follows. Let $\tilde{Q} \in \mathrm{L}$ be a cube for which $\phi(\tilde{Q})$ is an $I$-cube, and let $x_{0} \in \tilde{Q} \cap \partial|\widetilde{\mathrm{L}}|$. We set $\iota: \partial|\widetilde{\mathrm{L}}| \rightarrow \partial|\widetilde{\mathrm{L}}|$ be the unique lift of the identity map id: $\partial|\mathrm{L}| \rightarrow \partial|\mathrm{L}|$ satisfying

$$
S\left(\iota\left(x_{0}\right)\right)=\tilde{h}_{\rho}^{\prime}\left(S\left(x_{0}\right)\right) .
$$

After these preliminaries, we are now ready to define the extension of $\tilde{h}_{\rho}^{\prime}$. Let $\tilde{h}_{\rho}:|\widetilde{\mathrm{L}}| \rightarrow|\widetilde{\mathrm{L}}|$ be the unique map satisfying $\left.\tilde{h}_{\rho}\right|_{\partial|\widetilde{\mathrm{L}}|}=\iota,\left.\tilde{h}_{\rho}\right|_{\mid \widetilde{\mathrm{L}}^{\prime}}=\tilde{h}_{\rho}^{\prime}$, and which, for each $x \in \partial|\widetilde{\mathrm{L}}|$ affinely maps the segment $[x, S(x)]$ to the segment $\left[\iota(x), \tilde{h}_{\rho}^{\prime}(S(x))\right]$.

Before discussing the extension of $\tilde{h}_{\rho}$, we estimate its bilipschitz constant. Since $\tilde{h}_{\rho}^{\prime}$ is a lift of a realization of a rotation $\rho$, there exists a bijection $\tilde{\rho}: \widetilde{L^{\prime}} \rightarrow \widetilde{L^{\prime}}$ having $\tilde{h}_{\rho}^{\prime}$ as its realization. In fact, $\tilde{\rho}$ is a lift of $\rho$. The bijection $\tilde{\rho}$ is a translation in $\widetilde{\mathrm{L}}^{\prime}$ by $k_{\rho} \in \mathbb{Z}$ cubes of $\widetilde{\mathrm{L}}^{\prime}$, that is, $\tilde{\rho}\left(\widetilde{Q}^{\prime}\right)=\widetilde{Q}^{\prime}+k_{\rho} \sigma(\widetilde{\mathrm{L}}) e_{1}$ for each $\widetilde{Q}^{\prime} \in \widetilde{L}^{\prime}$.

Since $\sigma\left(\mathrm{L}^{\prime}\right)=\sigma\left(\widetilde{\mathrm{L}^{\prime}}\right)$ and $\operatorname{dist}\left(\partial|\mathrm{L}|,\left|\mathrm{L}^{\prime}\right|\right)=\operatorname{dist}\left(\partial|\widetilde{\mathrm{L}}|, \mid \widetilde{\mathrm{L}^{\prime}}\right)$, we have that the bilipschitz constant of $\tilde{h}_{\rho}$ depends only on the ratio $\sigma\left(\mathrm{L}^{\prime}\right) k_{\rho} / \operatorname{dist}\left(\partial|\mathrm{L}|,\left|\mathrm{L}^{\prime}\right|\right)$. Since $\sigma\left(\mathrm{L}^{\prime}\right) k_{\rho} \leq \operatorname{diam}\left(\left|\mathrm{L}^{\prime}\right|\right) \leq \sigma\left(\mathrm{L}^{\prime}\right)\left(\# \mathrm{~L}^{\prime}\right)$ and the pair $\left(\mathrm{L}, \mathrm{L}^{\prime}\right)$ is uniform, we conclude that the bilipschitz constant of $\tilde{h}_{\rho}$ is bounded from above by

$$
\frac{\sigma\left(\mathrm{L}^{\prime}\right) k_{\rho}}{\operatorname{dist}\left(\partial|\mathrm{L}|,\left|\mathrm{L}^{\prime}\right|\right)} \lesssim \frac{\sigma\left(\mathrm{L}^{\prime}\right)\left(\# \mathrm{~L}^{\prime}\right)}{\sigma\left(\mathrm{L}^{\prime}\right)}=\# \mathrm{~L}^{\prime} .
$$

We return now to the extension of $\tilde{h}_{\rho}$. Since both $\iota$ and $\tilde{h}_{\rho}^{\prime}$ are deck transformation for corresponding restrictions of $g_{\phi}$, we conclude that $\tilde{h}_{\rho}$ is a deck transformation of the covering map $g_{\phi}$, that is, $g_{\phi}=g_{\phi} \circ \tilde{h}_{\rho}$. Thus $\tilde{h}_{\rho}$ decends to a bilipschitz homeomorphism $h_{\rho}:|\mathrm{L}| \rightarrow|\mathrm{L}|$. This completes the construction of $h_{\rho}$. It remains to show the local bilipschitz estimate (3.1).

For the argument, denote $\iota=\left(\iota_{1}, \iota_{2}\right):|\widetilde{\mathrm{L}}| \rightarrow \mathbb{R} \times[0, \sigma(\mathrm{L})]^{2}$ the coordinate functions of $\iota$, and denote $\tilde{h}_{\rho}^{\prime}=\left(\left(\tilde{h}_{\rho}^{\prime}\right)_{1},\left(\tilde{h}_{\rho}^{\prime}\right)_{2}\right):\left|\widetilde{\mathrm{L}^{\prime}}\right| \rightarrow \mathbb{R} \times\left(v+\left[0, \sigma\left(\mathrm{L}^{\prime}\right)\right]^{2}\right)$ the coordinate functions of $\tilde{h}_{\rho}^{\prime}$.

Let now $R: \mathbb{R}^{3} \rightarrow \mathbb{R}^{3}$ be the involution $R_{+}$and suppose that the nested pair ( $\left.\mathrm{L}, \mathrm{L}^{\prime}\right)$ is symmetric with respect to $R$; the case of $R_{-}$is similar. Then $\{0\} \times \mathbb{R}^{2} \cap|\mathrm{L}|$ is the fixed point set of $\left.R\right|_{|\mathrm{L}|}$. We may assume that $g_{\phi}(\{0\} \times$ $\left.\mathbb{R}^{2} \cap|\widetilde{\mathrm{L}}|\right) \subset\{0\} \times \mathbb{R}^{2} \cap|\mathrm{L}|$.

Since $|\mathrm{L}|$ is $R$-symmetric, we observe that also the map $g_{\phi}$ is symmetric in the sense that $g_{\phi}(-t, w)=R\left(g_{\phi}(t, w)\right)$ for all $(t, w) \in \mathbb{R} \times[0, \sigma(\mathrm{L})]^{2}$. Thus, the lift $\tilde{R}:|\widetilde{\mathrm{L}}| \rightarrow|\widetilde{\mathrm{L}}|$ of $\left.R\right|_{|\mathrm{L}|}:|\mathrm{L}| \rightarrow|\mathrm{L}|$ under $g_{\phi}$ is the standard involution $(x, y, z) \mapsto(-x, y, z)$.

We conclude that

$$
\tilde{H}=\tilde{h}_{\rho} \circ \tilde{R} \circ \tilde{h}_{\rho}^{-1}:|\widetilde{\mathrm{L}}| \rightarrow|\widetilde{\mathrm{L}}|
$$

is therefore an involution with the following properties:

(1) on $\partial|\widetilde{\mathrm{L}}|, \tilde{H}$ is the map $(t, z) \mapsto \iota\left(-\iota_{1}(t, x), \iota_{2}(t, w)\right)$, 
(2) on $\left|\widetilde{\mathrm{L}^{\prime}}\right|, \tilde{H}$ is the map $(t, w) \mapsto \tilde{h}_{\rho}^{\prime}\left(-\left(\tilde{h}_{\rho}^{\prime}\right)_{1}(t, w),\left(\tilde{h}_{\rho}^{\prime}\right)_{2}(t, w)\right)$, and

(3) for each $p \in \partial|\mathrm{L}|, \tilde{H}$ is an affine map on the segment $[p, S(p)]$.

For points $p \in \partial|\widetilde{\mathrm{L}}|$, we further have the estimate

$$
|\tilde{H}(p)-\tilde{H}(S(p))| \leq 2 \sigma\left(\mathrm{L}^{\prime}\right) k_{\rho} .
$$

Thus $\tilde{H}$ is bilipschitz with a constant depending only on $\# L^{\prime}$.

Since

$$
\begin{aligned}
g_{\phi} \circ \tilde{h}_{\rho} \circ \tilde{R} \circ \tilde{h}_{\rho}^{-1} & =h_{\rho} \circ g_{\phi} \circ \tilde{R} \circ \tilde{h}_{\rho}^{-1} \\
& =h_{\rho} \circ R \circ g_{\phi} \circ \tilde{h}_{\rho}^{-1}=h_{\rho} \circ R \circ h_{\rho}^{-1} \circ g_{\phi},
\end{aligned}
$$

we conclude that $h_{\rho} \circ R \circ h_{\rho}^{-1}$ satisfies the bilipschitz estimate (3.1).

\section{BING'S WILD INVOLUTION}

We begin now the construction of a cubical version of Bing's wild involution. The involution is based on the construction of a defining sequence for a decomposition in $\mathbb{S}^{3}$ and shrinkability of this decomposition. The wild Cantor set obtained by this construction is called Bing's double. We recall first this topological part of the construction and then discuss the involution.

We refer to Daverman's book [14] for the terminology related to decomposition spaces. We merely recall that the decomposition space $\mathbb{R}^{3} / E$ associated to a compact set $E \subset \mathbb{R}^{3}$ is the quotient space $\mathbb{R}^{3} / \sim$, where $\sim$ is the minimal equivalence relation for which $x \sim y$ if and only if the points $x$ and $y$ in $\mathbb{R}^{3}$ belong to the same component of $E$.

Remark. Although the statement of Theorem 1.1 is for involutions of $\mathbb{S}^{3}$, we work in $\mathbb{R}^{3}$ to simplify the notation. Note that the constructions of the defining sequences take place in a solid 3-torus in $\mathbb{R}^{3}$ and hence the wild involutions $\mathbb{R}^{3} \rightarrow \mathbb{R}^{3}$ we construct, extend naturally to involutions of $\mathbb{S}^{3}$ fixing the north pole $e_{4}$ after identification of $\mathbb{R}^{3}$ with $\mathbb{S}^{3} \backslash\left\{e_{4}\right\}$ by the stereographic projection.

4.1. The Set-up. For the rest of the discussion, we fix $R: \mathbb{R}^{3} \rightarrow \mathbb{R}^{3}$ to be either the orientation reversing involution

$$
R:(x, y, z) \mapsto(-x, y, z)
$$

considered by Bing [5] or the orientation preserving involution

$$
R:(x, y, z) \mapsto(-x,-y, z)
$$

considered by Montgomery and Zippin [25]. Having this choice in mind, we may recall Bing's construction for a shrinkable decomposition $\widetilde{B}$ of $\mathbb{R}^{3}$ invariant under this involution.

The defining sequence $\left(\widetilde{X}_{k}\right)$ for the decomposition $\widetilde{B}$ is given as follows. First, let $\left(T_{w}\right)_{w}$ be a tree of solid 3-tori as in [7, Fig. 7], where $w$ is a finite word in letters $\{+,-\}$; the solid tori corresponding the empty word $\varnothing$ we denote $T_{0}$. We refer to $[5,7]$ for a description of the linking; the tori $T_{w+}$ and $T_{w-}$ are said to 'hook elbows'. As in $[5,7]$ and [25], we assume that each solid torus $T_{w}$ is symmetric with respect to the fixed involution $R$, that is, for each word $w$, the map $\left.R\right|_{T_{w}}: T_{w} \rightarrow T_{w}$ is a well-defined bijection. 
For each $k \in \mathbb{N}$, we set $\widetilde{X}_{k}$ to be the union of all solid tori $T_{w}$ for words $w$ of length $k$. Let now $\widetilde{\mathrm{B}}$ be the decomposition of $\mathbb{R}^{3}$ for which the non-trivial elements are the components of the intersection

$$
\widetilde{X}=\bigcap_{k \geq 1} \tilde{X}_{k}
$$

the set $\widetilde{X}$ is a compact set consisting of line segements indexed by a Cantor set $\widetilde{X} \cap\left(\{0\} \times \mathbb{R}^{2}\right)$ in the $y z$-plane $\{0\} \times \mathbb{R}^{2}$; see $[5]$.

Bing shows the shrinkability of the decomposition $\widetilde{B}$ by a delicate folding procedure of the tori $T_{w}$; see [7, Figures 9-12] and [7, pp. 491-492] for a careful description of the foldings which yield the shrinkability of the decomposition $\widetilde{B}$.

4.1.1. Heuristical description of the cubical defining sequence. We describe now Bing's construction in our terminology. In what follows, we fix a tree $\left(\mathrm{L}_{w}\right)_{w}$ of cubical loops for which the tree of solid tori $\left(\left|\mathrm{L}_{w}\right|\right)_{w}$ is symmetric with respect to $R$ and topologically equivalent to Bing's construction. Bing's folding maps correspond to a choice of a family of twist maps $\left\{\left(\left|\mathrm{L}_{w}\right|,\left|\mathrm{L}_{w}^{\prime}\right|\right) \rightarrow\right.$ $\left.\left(\left|\mathbf{L}_{w}\right|,\left|\mathbf{L}_{w}^{\prime}\right|\right)\right\}_{w}$ associated to the tree $\left(\mathrm{L}_{w}\right)_{w}$ and cubical loops $\mathbf{L}_{w}^{\prime}$ nested into loops $\mathrm{L}_{w}$, respectively.

Whereas a careful choice of loops $L_{w}$ and $L_{w}^{\prime}$ is crucial for our proof, the particular choices of the angles of associated twist maps are not relevant for the regularity considerations. Therefore, in what follows, we discuss mainly the cubical loops and the generic bilipschitz properties of the twist maps $\left(\left|\mathrm{L}_{w}\right|,\left|\mathrm{L}_{w}^{\prime}\right|\right) \rightarrow\left(\left|\mathrm{L}_{w}\right|,\left|\mathrm{L}_{w}^{\prime}\right|\right)$.

Let now, for each $k \geq 1$,

$$
X_{k}=\bigcup_{|w|=k}\left|\mathrm{~L}_{w}\right|
$$

and let $\mathrm{B}$ be the decomposition associated to sequence $\left(X_{k}\right)$. Finally, let $X$ be intersection

$$
X=\bigcap_{k \geq 1} X_{k}
$$

and let $\phi: \mathbb{R}^{3} \rightarrow \mathbb{R}^{3}$ be the monotone map associated to the decomposition $\mathrm{B}$, that is, $\left.\phi\right|_{\mathbb{R}^{3} \backslash X}: \mathbb{R}^{3} \backslash X \rightarrow \mathbb{R}^{3} \backslash \phi(X)$ is a homeomorphism and, for each non-trivial element $C$ of $\mathrm{B}$, the image $\phi(C)$ is a point.

Since each non-trivial element of $\mathrm{B}$ is invariant under $R$, the reflection $R$ decends with respect to $\phi$, that is, there exists a (wild) involution $f_{\phi}: \mathbb{R}^{3} \rightarrow$ $\mathbb{R}^{3}$ the (wild) involution satisfying $f_{\phi} \circ \phi=\phi \circ R$.

The purpose of the following sections is to show that, for each $p \in[1,2)$, we may choose such a monotone map $\phi$ that the involution $f_{\phi}$ belongs to the Sobolev space $W^{1, p}$.

4.2. Cubical version of Bing's construction. We fix now a particular cubical configuration of loops. Apart from terminology, the following conditions are as in $[5,7]$. 
4.2.1. Splitting a loop. In the construction of Bing's double, two cubical loops are embedded into a cubical loop in each stage. These new loops are properly embedded into arcs splitting the larger loop. For this reason we introduce this last bit of terminology.

A loop $L^{\prime}$ is properly nested into an arc $\mathrm{A}$ if

(1) $\left|\mathrm{L}^{\prime}\right| \subset \operatorname{int}|\mathrm{A}|$,

(2) $I$-cubes of A contain only $I$-cubes of $\mathrm{L}^{\prime}$,

(3) each terminal cube of A contains exactly two corners of $L^{\prime}$, and

(4) each corner of $A$ contains exactly two corners of $L^{\prime}$ and these three corners have the same symmetry plane.

Let $L$ be a cubical loop. A pair $A^{+}$and $A^{-}$of arcs is a splitting of $L$ if $A^{-} \cup A^{+}=L$ and $A^{-} \cap A^{+}=\operatorname{Terminal}\left(A^{-}\right)$; note that Terminal $\left(A^{+}\right)=$ Terminal $\left(A^{-}\right)$; see Figure 8 for an illustration.

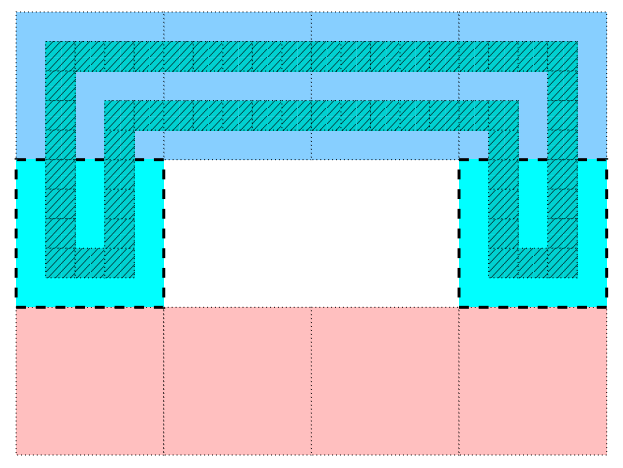

Figure 8. Splitting of a loop.

4.2.2. Cubical loops. Let $\left(\mathrm{L}_{w}\right)_{w}$ be a tree of nested loops, which are invariant under $R$, as in the initial configuration of Bing's double. We may also assume that $L_{0}$ is a model loop having two long sides and two short sides and $\# \mathrm{~L}_{0} \geq 12$, say; see Figure 9 .

For each $k$, let $r_{k}$ be the common side length of cubes in loops $\mathrm{L}_{w}$ for all words $w$ of length $k$. We fix the sequence $\left(r_{k}\right)$ later.

For each word $w$, let $\mathrm{A}_{w}^{+}$and $\mathrm{A}_{w}^{-}$be arcs splitting $\mathrm{L}_{w}$ and let $\mathrm{L}_{w+}$ and $\mathrm{L}_{w-}$ be loops properly embedded into $\mathrm{A}_{w}^{+}$and $\mathrm{A}_{w}^{-}$in such a way that the solid 3 -tori $\left|\mathrm{L}_{w+}\right|$ and $\left|\mathrm{L}_{w-}\right|$ are linked in $\left|\mathrm{L}_{w}\right|$ but not in $\mathbb{R}^{3}$. Due to the symmetry of $\mathrm{L}_{w}$ under $R$, we may assume that $\operatorname{arcs} \mathrm{A}_{w}^{ \pm}$are symmetric with respect to $R$ in the sense that $\mathrm{A}_{w}^{ \pm} \rightarrow \mathrm{A}_{w}^{ \pm}, Q \mapsto R(Q)$, is a well-defined bijection. Note that, we do not require that $\mathrm{A}^{+}$and $\mathrm{A}^{-}$have the same number of cubes.

For each word $w$, let $\mathrm{L}_{w}^{\prime}$ be a loop nested in $\mathrm{L}_{w}$ of side length $\sigma\left(\mathrm{L}_{w}^{\prime}\right)=$ $\sigma\left(\mathrm{L}_{w}\right) / 3$ and satisfying dist $\left(\left|\mathrm{L}_{w}^{\prime}\right|, \partial\left|\mathrm{L}_{w}\right|\right)=s\left(\mathrm{~L}_{w}^{\prime}\right)$. We split $\mathrm{L}_{w}^{\prime}$ into two arcs $\mathrm{A}_{w}^{+}$and $\mathrm{A}_{w}^{-}$for which the terminal cubes of $\mathrm{A}_{w}^{+}$and $\mathrm{A}_{w}^{-}$are contained in the terminal cubes of the arcs in the previous level, that is, $A_{w+}^{\prime} \cap A_{w-}^{\prime} \subset$ $\mathrm{A}_{v+}^{\prime} \cap \mathrm{A}_{v-}^{\prime}$, where $v$ is the unique word for which either $w=v+$ or $w=v-$. We also place loops $\mathrm{L}_{w+}$ and $\mathrm{L}_{w-}$, contained in $\left|\mathrm{L}_{w}\right|$, into $\operatorname{arcs} \mathrm{A}_{w}^{+}$and $\mathrm{A}_{w}^{-}$ so that they are properly nested $\mathrm{A}_{w}^{+}$and $\mathrm{A}_{w}^{-}$, respectively. We also require that $\sigma\left(\mathrm{L}_{w \pm}\right) \leq \sigma\left(\mathrm{L}_{w}^{\prime}\right) / 5$. 




FiguRE 9. Nested loops $\mathrm{L}_{0}^{\prime}$ and $\mathrm{L}_{0}$ and loops $\mathrm{L}_{+}$and $\mathrm{L}_{-}$ properly embedded in $\operatorname{arcs} A_{+}$and $A_{-}$, respectively, splitting the loop $\mathrm{L}_{0}^{\prime}$.

4.2.3. The monotone map. The monotone map $\phi: \mathbb{R}^{3} \rightarrow \mathbb{R}^{3}$ is defined as follows. As the first step, we observe that, for a word $w$, we may choose Bing's folding map in $\left|\mathrm{L}_{w}\right|$ to be a twist map $\theta_{w}:\left|\mathrm{L}_{w}\right| \rightarrow\left|\mathrm{L}_{w}\right|$ associated to a (particular) rotation $\rho_{w}: \mathrm{L}_{w}^{\prime} \rightarrow \mathrm{L}_{w}^{\prime}$; see [7].

As the second step, we set, for each $k \in \mathbb{N}, \theta_{k}: X_{k} \rightarrow X_{k}$ to be the homeomorphism satisfying $\left.\theta_{k}\right|_{\mathbb{R}^{3} \backslash X_{k}}=$ id and $\left.\theta_{k}\right|_{\left|\mathbf{L}_{w}\right|}=\theta_{w}$ for each word $w$ of length $k$.

As the third step, we define $\phi: \mathbb{R}^{3} \rightarrow \mathbb{R}^{3}$ by

$$
\phi=\lim _{k \rightarrow \infty} \theta_{1} \circ \cdots \circ \theta_{k} .
$$

Since $\phi$ is a uniform limit of a sequence of homeomorphisms, it is a monotone map.

The wild involution $f: \mathbb{R}^{3} \rightarrow \mathbb{R}^{3}$ is now the unique involution satisfying

$$
f \circ \phi=\phi \circ R \text {. }
$$

To simplify notation, we write also

$$
\psi_{k}=\theta_{1} \circ \cdots \circ \theta_{k}: \mathbb{R}^{3} \rightarrow \mathbb{R}^{3}
$$

and

$$
f_{k}=\psi_{k} \circ R \circ \psi_{k}^{-1}: \mathbb{R}^{3} \rightarrow \mathbb{R}^{3}
$$

for each $k \in \mathbb{N}$. Then $f_{k} \rightarrow f$ uniformly as $k \rightarrow \infty$. Note that, locally, each $\psi_{k}$ is a composition of twist maps and hence $\psi_{k}$ is a bilipschitz homeomorphism for each $k \in \mathbb{N}$.

4.2.4. Twisted tori $\mathrm{T}_{w}$ and associated functions. Locally in $\mathbb{R}^{3} \backslash X$, the map $\phi$ is a finite composition of twist maps. Thus there exists a family $\left(\mathbf{T}_{w}\right)_{w}$ of nested cubical tori for which $\left|\mathrm{T}_{w}\right|=\psi_{k}\left(\left|\mathrm{~L}_{w}\right|\right)$ and isomorphisms $\beta_{w}: \mathrm{L}_{w} \rightarrow$ $\mathrm{T}_{w}$ for which $f_{|w|} \circ \beta_{w}=\beta_{w} \circ R$ for each word $w$. More precisely, for each $w$ there exists a cubical loop $\mathrm{T}_{w}^{\prime}$ properly nested in $\mathrm{T}_{w}$ for which $\left|\mathrm{T}_{w}\right| \backslash\left|\mathrm{T}_{w}^{\prime}\right|=$ 
$\psi_{k}\left(\left|\mathrm{~L}_{w}\right| \backslash\left|\mathrm{L}_{w}^{\prime}\right|\right)$ and the loops $\mathrm{T}_{w+}$ and $\mathrm{T}_{w-}$ are nested in $\operatorname{arcs} \mathrm{A}_{w+}^{\top}$ and $\mathrm{A}_{w-}^{\top}$ splitting $\mathrm{T}_{w}$.

We also denote

$$
Z_{k}=\bigcup_{|w|=k}\left|\mathrm{~T}_{w}\right| \quad \text { and } \quad Z_{k}^{\prime}=\bigcup_{|w|=k}\left|\mathrm{~T}_{k}^{\prime}\right|
$$

for each $k \in \mathbb{Z}_{+}$. We also set

$$
Z_{0}=\mathbb{R}^{3} \backslash Z_{1} \quad \text { and } \quad Z=\bigcap_{k \in \mathbb{Z}_{+}} Z_{k} .
$$

Then, in particular,

$$
Z_{k}=\psi_{k}\left(X_{k}\right) \text { for each } k \geq 1 \text { and } Z=\phi(X) .
$$

We also define two auxiliary functions related to cubical nested tori $\mathbf{T}_{w}$. The level $\ell(x)$ of $x \in \mathbb{R}^{3}$ with respect to $\left(\mathrm{T}_{w}\right)_{w}$ is

$$
\ell(x)= \begin{cases}k, & \text { if } x \in Z_{k} \backslash Z_{k+1} \\ 0, & \text { otherwise, }\end{cases}
$$

and the corner index $\eta(x)$ of $x \in \mathbb{R}^{3}$ with respect to $\left(\mathbf{T}_{w}\right)_{w}$ is

$$
\eta(x)=\#\left\{w: x \in \operatorname{Corner}\left(\mathbf{T}_{w}\right)\right\} \in \mathbb{N} \cup\{\infty\} .
$$

4.2.5. Local bilipschitz constant of $f_{k}$ on $Z_{k} \backslash Z_{k+1}$ for $k \geq 0$. Let $k \geq 1$ and $x \in Z_{k} \backslash Z_{k+1}$. Since $\psi_{k-1}$ is a composition of twist maps, we have, by the proof of Lemma 2.6, that

$$
\operatorname{Lip}\left(\psi_{k-1}\right) \leq L_{\text {inner }}^{\eta(x)},
$$

where $L_{\text {inner }}$ is universal. On the other hand, by Lemma 3.3, we have that

$$
\operatorname{Lip}\left(\theta_{k} \circ R \circ \theta_{k}^{-1}\right) \leq L_{\diamond} \cdot 3 \cdot \# \mathrm{~T}_{w}^{\prime}=3 L_{\diamond}\left(\# \mathrm{~L}_{w}^{\prime}\right) \leq 9 L_{\diamond}\left(\# \mathrm{~L}_{w}\right) .
$$

Thus

$$
\operatorname{Lip} f_{k}=\operatorname{Lip}\left(\psi_{k-1} \circ\left(\theta_{k} \circ R \circ \theta_{k}^{-1}\right) \circ \psi_{k-1}^{-1}\right) \leq L_{1} L_{\text {inner }}^{2 \eta(x)} / r_{\ell(x)}
$$

near $x$, where $L_{1}$ is universal.

\section{Proof of Theorem 1.1}

Let $p \in[1,2)$. It remains to show that we may choose side lengths of cubes in the loop $L_{w}$ in such a way that the reflection $f: \mathbb{R}^{3} \rightarrow \mathbb{R}^{3}$ induced by the reflection $R$ is in the Sobolev space $W^{1, p}\left(\mathbb{R}^{3}, \mathbb{R}^{3}\right)$.

For each $k \in \mathbb{N}$, let

$$
m_{k}=\frac{k}{2-p}
$$

and, for each word $w$ of length $k$, we set the cube size $r_{k}$ of $\mathbf{L}_{w}$ to be

$$
r_{k}=\min \left\{3^{-m_{k}}, r_{k-1} / 15,\left(10 L_{\text {inner }}^{p}\right)^{-k}\right\} ;
$$

here the condition $r_{k} \leq r_{k-1} / 15$ guarantees that conditions $\sigma\left(\mathrm{L}_{w+}\right)=$ $\sigma\left(\mathrm{L}_{w-}\right) \leq \sigma\left(\mathrm{L}_{w}^{\prime}\right) / 5 \leq \sigma\left(\mathrm{L}_{w}\right) / 15$ are satisfied.

Let now the monotone map $\phi: \mathbb{R}^{3} \rightarrow \mathbb{R}^{3}$ and the involution $f: \mathbb{R}^{3} \rightarrow \mathbb{R}^{3}$ be as in the previous section. 
By (4.1), the involution $f: \mathbb{R}^{3} \rightarrow \mathbb{R}^{3}$ is locally $L(x)$-Lipschitz for each $x \in \mathbb{R}^{3} \backslash \phi(X)$, where

Hence

$$
L(x)=L_{1} L_{\text {inner }}^{2 \eta(x)} / r_{\ell(x)} .
$$

$$
|D f(x)| \leq L(x)
$$

for almost every $x \in \mathbb{R}^{3} \backslash \phi(X)$.

By [7], the number of corners of $T_{w}$ is at most double the corners of $\mathbf{L}_{w}$ and the cubical loop $\mathrm{L}_{w}$ has $4^{|w|}$ corners. Thus, for each $k \in \mathbb{N}$, we have also the estimate

$$
\left|\left\{x \in Z_{k}: \eta(x)>0\right\}\right| \lesssim 4^{k+1} r_{k}^{3} \leq 4^{k+1}\left(10 L_{\text {inner }}^{p}\right)^{-k} \lesssim\left(2 L_{\text {inner }}^{p}\right)^{-k}
$$

for the Lebesgue measure of the set of those points of $Z_{k}$, which are contained in the corners on some previous level. We conclude that

$$
\int_{\left\{x \in Z_{k} \backslash Z_{k+1}: \eta(x)>0\right\}}|D f|^{p} \lesssim\left(L_{\text {inner }}^{k}\right)^{p}\left(2 L_{\text {inner }}^{p}\right)^{-k}=2^{-k} .
$$

We estimate now the $L^{p}$-norm of $|D f|$ on the sets

$$
Y_{k}=\left\{x \in Z_{k} \backslash Z_{k+1}: \eta(x)=0\right\} .
$$

Since $Z_{k}$ is a pair-wise disjoint union of $2^{k}$ loops consisting at most $2 / r_{k}$ cubes of side length $r_{k}$, we have the estimate

$$
\left|Z_{k}\right| \leq 2^{k}\left(2 / r_{k}\right) r_{k}^{3}=2^{k+1} r_{k}^{2}
$$

for the Lebesgue measure $\left|Z_{k}\right|$ of $Z_{k}$. Thus

$$
\int_{Y_{k}}|D f|^{p} \mathrm{~d} x \leq\left(L_{1} / r_{k}\right)^{p}\left|Z_{k}\right| \leq L_{1} 2^{k+1} r_{k}^{2-p} \leq L_{1} 2^{k+1} 3^{-k}=2 L_{1}(2 / 3)^{k} .
$$

We conclude that

$$
\int_{Z_{0} \backslash Z}|D f|^{p} \mathrm{~d} x=\sum_{k \geq 0} \int_{Z_{k} \backslash Z_{k+1}}|D f|^{p} \mathrm{~d} x \leq \sum_{k \geq 0} 3 L_{1}(2 / 3)^{k}=9 L_{1} .
$$

Since the Cantor set $Z$ has Lebesgue 3-measure zero, we have that $|D f|$ is in $L^{p}\left(Z_{0}, \mathbb{R}^{3}\right)$.

It remains now to verify that $f$ is weakly differentiable. Recall that

$$
f_{k}=\psi_{k}^{-1} \circ R \circ \psi_{k} \rightarrow f
$$

as $k \rightarrow \infty$. Each $f_{k}$ is Lipschitz and, by the previous calculation, $\left(f_{k}\right)$ is a Cauchy sequence in $W^{1, p}\left(\mathbb{R}^{3}, \mathbb{R}^{3}\right)$. Since the sequence $\left(f_{k}\right)$ converges to $f$ locally uniformly, we conclude that $f \in W^{1, p}\left(\mathbb{R}^{3}, \mathbb{R}^{3}\right)$. This completes the proof.

\section{REFERENCES}

[1] S. S. Antman. Nonlinear problems of elasticity, volume 107 of Applied Mathematical Sciences. Springer, New York, second edition, 2005.

[2] S. Armentrout. Concerning cellular decompositions of 3-manifolds that yield 3manifolds. Trans. Amer. Math. Soc., 133:307-332, 1968.

[3] J. M. Ball. Convexity conditions and existence theorems in nonlinear elasticity. Arch. Rational Mech. Anal., 63(4):337-403, 1976/77.

[4] J. M. Ball. Singularities and computation of minimizers for variational problems. In Foundations of computational mathematics (Oxford, 1999), volume 284 of London Math. Soc. Lecture Note Ser., pages 1-20. Cambridge Univ. Press, Cambridge, 2001. 
[5] R. H. Bing. A homeomorphism between the 3-sphere and the sum of two solid horned spheres. Ann. of Math. (2), 56:354-362, 1952.

[6] R. H. Bing. Decompositions of $E^{3}$. In Topology of 3-manifolds and related topics (Proc. The Univ. of Georgia Institute, 1961), pages 5-21. Prentice-Hall. Englewood Cliffs, N.J., 1962.

[7] R. H. Bing. Shrinking without lengthening. Topology, 27(4):487-493, 1988.

[8] S. Bochner. Compact groups of differentiable transformations. Ann. of Math. (2), 46:372-381, 1945.

[9] L. E. J. Brouwer. Über die periodischen Transformationen der Kugel. Math. Ann., 80(1):39-41, 1919.

[10] M. Brown. A proof of the generalized Schoenflies theorem. Bull. Amer. Math. Soc., $66: 74-76,1960$.

[11] M. Brown. The monotone union of open $n$-cells is an open $n$-cell. Proc. Amer. Math. Soc., 12:812-814, 1961.

[12] D. Campbell, S. Hencl, and V. Tengvall. Approximation of $W^{1, p}$ Sobolev homeomorphism by diffeomorphisms and the signs of the Jacobian. Adv. Math., 331:748-829, 2018.

[13] P. G. Ciarlet. Mathematical elasticity. Vol. I, volume 20 of Studies in Mathematics and its Applications. North-Holland Publishing Co., Amsterdam, 1988. Three-dimensional elasticity.

[14] R. J. Daverman. Decompositions of manifolds, volume 124 of Pure and Applied Mathematics. Academic Press Inc., Orlando, FL, 1986.

[15] D. Drasin and P. Pankka. Sharpness of Rickman's Picard theorem in all dimensions. Acta Math., 214(2):209-306, 2015.

[16] M. H. Freedman and R. Skora. Strange actions of groups on spheres. J. Differential Geom., 25(1):75-98, 1987.

[17] P. Hajłasz and P. Koskela. Sobolev meets Poincaré. C. R. Acad. Sci. Paris Sér. I Math., 320(10):1211-1215, 1995.

[18] D. H. Hamilton. QC Riemann mapping theorem in space. In Complex analysis and dynamical systems III, volume 455 of Contemp. Math., pages 131-149. Amer. Math. Soc., Providence, RI, 2008.

[19] J. Heinonen and S. Semmes. Thirty-three yes or no questions about mappings, measures, and metrics. Conform. Geom. Dyn., 1:1-12 (electronic), 1997.

[20] J. Heinonen and J.-M. Wu. Quasisymmetric nonparametrization and spaces associated with the Whitehead continuum. Geometry \& Topology, 14(2):773-798, 2010.

[21] S. Hencl and A. Pratelli. Diffeomorphic approximation of $W^{1,1}$ planar Sobolev homeomorphisms. J. Eur. Math. Soc. (JEMS), 20(3):597-656, 2018.

[22] S. Hencl and B. Vejnar. Sobolev homeomorphism that cannot be approximated by diffeomorphisms in $W^{1,1}$. Arch. Ration. Mech. Anal., 219(1):183-202, 2016.

[23] T. Iwaniec, L. V. Kovalev, and J. Onninen. Diffeomorphic approximation of Sobolev homeomorphisms. Arch. Ration. Mech. Anal., 201(3):1047-1067, 2011.

[24] T. Iwaniec and J. Onninen. Monotone Sobolev mappings of planar domains and surfaces. Arch. Ration. Mech. Anal., 219(1):159-181, 2016.

[25] D. Montgomery and L. Zippin. Examples of transformation groups. Proc. Amer. Math. Soc., 5:460-465, 1954.

[26] J. W. Morgan and H. Bass, editors. The Smith conjecture, volume 112 of Pure and Applied Mathematics. Academic Press, Inc., Orlando, FL, 1984. Papers presented at the symposium held at Columbia University, New York, 1979.

[27] P. Pankka and V. Vellis. Quasiconformal non-parametrizability of almost smooth spheres. Selecta Mathematica, 23(2):1121-1151, 2017.

[28] P. Pankka and J.-M. Wu. Geometry and quasisymmetric parametrization of Semmes spaces. Rev. Mat. Iberoamericana, 30(3):893-960, 2014.

[29] S. Semmes. Good metric spaces without good parameterizations. Rev. Mat. Iberoamericana, 12(1):187-275, 1996.

[30] L. C. Siebenmann. Approximating cellular maps by homeomorphisms. Topology, 11:271-294, 1972.

[31] P. A. Smith. Transformations of finite period. II. Ann. of Math. (2), 40:690-711, 1939. 
[32] F. Waldhausen. Über Involutionen der 3-Sphäre. Topology, 8:81-91, 1969.

[33] J. W. T. Youngs. Homeomorphic approximations to monotone mappings. Duke Math. J., 15:87-94, 1948.

Department of Mathematics, Syracuse University, Syracuse, NY 13244, USA and Department of Mathematics and Statistics, P.O.Box 35 (MaD) FI-40014 UNIVERSiTy OF JyVÄSKYLÄ, Finland

Email address: jkonnine@syr.edu

Department of Mathematics and Statistics, P.O. Box 68 (Pietari Kalmin Katu 5), FI-00014 University of Helsinki, Finland

Email address: pekka.pankka@helsinki.fi 